\title{
Deterioration of pulmonary hypertension and pleural effusion with bosutinib following dasatinib lung toxicity
}

\begin{abstract}
To the Editor:
Tyrosine kinase inhibitors (TKIs) targeting BCR/ABL such as imatinib, nilotinib, dasatinib, bosutinib and ponatinib have revolutionised the management of patients with chronic myeloid leukaemia (CML) [1]. Pleural effusions and pulmonary arterial hypertension $(\mathrm{PAH})$ have been reported in patients treated with these agents [2-4]. These side-effects are more frequently observed with dasatinib use, with partial or complete reversibility after drug withdrawal [3]. Bosutinib is a second-generation TKI prescribed in case of intolerance or resistance to imatinib, nilotinib or dasatinib. In the present report, we describe two cases of worsening of dasatinib-induced $\mathrm{PAH}$ and two cases of severe pleural effusions, suggesting overlapping pulmonary toxicity of bosutinib and dasatinib.
\end{abstract}

Patient 1, a 44-year-old Caucasian woman with CML, was successively treated with hydroxycarbamide, imatinib, nilotinib and dasatinib. After 3 years on dasatinib, she complained of exertional dyspnoea, New York Heart Association functional class (NYHA FC) II. Echocardiography showed a dilated right ventricle and an estimated systolic pulmonary artery pressure (PAP) of $80 \mathrm{mmHg}$. Right heart catheterisation demonstrated pre-capillary pulmonary hypertension (mean PAP $34 \mathrm{mmHg}$, pulmonary capillary wedge pressure (PCWP) $9 \mathrm{mmHg}$, cardiac index (CI) $3.8 \mathrm{~L} \cdot \mathrm{min}^{-1} \cdot \mathrm{m}^{-2}$ and pulmonary vascular resistance (PVR) 3.7 Wood Units (WU)). A diagnosis of PAH induced by dasatinib was established and the patient was switched to bosutinib (500 mg once daily). After an initial clinical improvement, the patient's condition deteriorated rapidly after 2 months on bosutinib (NYHA FC IV with right heart failure and pericardial effusion). Right heart catheterisation confirmed haemodynamic deterioration (mean PAP $50 \mathrm{mmHg}$, CI $3.1 \mathrm{~L} \cdot \mathrm{min}^{-1} \cdot \mathrm{m}^{-2}$, PCWP $6 \mathrm{mmHg}$ and PVR $8.45 \mathrm{WU}$ ). Bosutinib was discontinued and bosentan initiated with a rapid improvement (NYHA FC I). At 6 months, 6-min walk distance was $474 \mathrm{~m}$ and right heart catheterisation showed haemodynamic improvement (mean PAP $27 \mathrm{mmHg}$, PCWP $5 \mathrm{mmHg}$, CI $3 \mathrm{~L} \cdot \mathrm{min}^{-1} \cdot \mathrm{m}^{-2}$ and PVR $4.1 \mathrm{WU}$ ).

Patient 2, a 48-year-old Caucasian woman with CML, was treated with imatinib during 2 years followed by dasatinib. After 2 years on dasatinib, she started complaining of exertional dyspnoea and pulmonary hypertension was suspected on echocardiography. Combined pre- and post-capillary pulmonary hypertension was diagnosed (mean PAP $45 \mathrm{mmHg}$, systolic PAP $75 \mathrm{mmHg}$, diastolic PAP $32 \mathrm{mmHg}$, right atrial pressure $9 \mathrm{mmHg}$, PCWP $19 \mathrm{mmHg}$, CI $3.1 \mathrm{~L} \cdot \mathrm{min}^{-1} \cdot \mathrm{m}^{-2}$ and PVR 4.6 WU). Dasatinib was switched to nilotinib and then imatinib. In October 2012, 7 months after dasatinib withdrawal, right heart catheterisation showed persistence of PAH and tadalafil was initiated. In June 2013, the patient had mild pre-capillary pulmonary hypertension (mean PAP $30 \mathrm{mmHg}$, PCWP $8 \mathrm{mmHg}$, CI $3.9 \mathrm{~L} \cdot \mathrm{min}^{-1} \cdot \mathrm{m}^{-2}$ and PVR 2.7 WU). In November 2013, imatinib was switched to bosutinib (500 mg once daily) because of unsatisfactory haematological response. After 4 months of bosutinib, the patient deteriorated (NYHA FC III) and right heart catheterisation documented a marked haemodynamic deterioration (mean PAP $47 \mathrm{mmHg}$, PCWP $15 \mathrm{mmHg}$, CI $3.4 \mathrm{~L} \cdot \mathrm{min}^{-1} \cdot \mathrm{m}^{-2}$ and PVR $4.8 \mathrm{WU}$ ). As there were limited therapeutic alternatives, bosutinib was continued with a dose reduction at $300 \mathrm{mg}$ once daily. A persistent haemodynamic aggravation was confirmed in March 2016 (mean PAP $53 \mathrm{mmHg}$, PCWP $13 \mathrm{mmHg}$, CI 2.6 L $\cdot \mathrm{min}^{-1} \cdot \mathrm{m}^{-2}$ and PVR 7.7 WU). Ambrisentan was combined with tadalafil and bosutinib was stopped.

Patient 3, a 79-year-old Caucasian woman with CML receiving imatinib since 2014, was switched to dasatinib (100 mg once daily) in August 2014 because of a skin rash. At this time, echocardiography was normal. In March 2015, the patient started complaining of exertional dyspnoea revealing moderate bilateral pleural effusions. Dasatinib was continued at the same dose and finally switched to bosutinib in July 2015 because of persistence of minimal pleural effusion despite diuretics. One month later, dyspnoea worsened due to an abundant bilateral lymphocyte-rich pleural exudate. Management included bosutinib withdrawal, diuretic and prednisolone $(30 \mathrm{mg})$. The patient's condition improved rapidly and corticosteroid was gradually discontinued without relapse.

Patient 4, a 81-year-old Caucasian woman with CML diagnosed in 2005, was treated with imatinib for 2 years followed by dasatinib. In March 2009, dasatinib was switched to nilotinib because of pleural and pericardial effusion, which rapidly disappeared after dasatinib withdrawal. In October 2014, treatment was changed to bosutinib (400 mg once daily). Six months after, exertional dyspnoea revealed mild bilateral pleural effusions. Bosutinib was continued and 6 months later the clinical condition deteriorated with 
severe bilateral pleural and pericardial effusions. The analysis of the pleural fluid showed lymphocyte-rich pleural exudate. Bosutinib was discontinued and the pleural effusion rapidly regressed.

We report four cases of pulmonary vascular and pleural complications of bosutinib, a novel TKI approved for patients with CML. As a result of the structural conservation of the ATP-binding pocket between diverse protein kinases, TKI selectivity is often a critical issue for the development of these compounds and explains why most TKIs have inhibitory actions against a wide variety of kinases. Since multiple signalling pathways are involved in most tumours, the possibility to inhibit several kinases simultaneously sometimes represents an advantage. However, overall toxicity of TKIs may also be related to the nonselective inhibition of several kinases. Until now, pulmonary side-effects, including pleural effusions and $\mathrm{PAH}$, have been more frequently reported with dasatinib than with other TKIs. Indeed, dasatinib-induced exudative pleural effusion is a frequent adverse event ranging from $15 \%$ to $35 \%$ [2, 3, 5-7]. Although the known incidence of bosutinib-induced pleural effusion is considered to be rare $[8,9]$, we report two cases of exudative lymphocytic pleural effusions that rapidly regressed after bosutinib withdrawal. Dasatinib may also induce $\mathrm{PAH}$ with an estimated lowest incidence of PAH of $0.45 \%$ [3]. We recently demonstrated that dasatinib causes pulmonary vascular damage through mitochondrial reactive oxygen species production in a dose-dependent manner, leading to increased susceptibility to develop PAH [10]. Unique to dasatinib-associated PAH (e.g. as compared with anorectic agents), the pulmonary vascular disease improves in the majority of patients upon withdrawal of dasatinib [3]. As our two cases of bosutinib-associated PAH occurred in patients with prior dasatinib-induced $\mathrm{PAH}$, we cannot exclude that these cases were potentiated or facilitated by the prior use of dasatinib. This hypothesis is in accordance with our recent results showing that dasatinib may increase the risk of developing pulmonary hypertension in animal models in the presence of a second "hit" (monocrotaline or chronic hypoxia) [10].

A case of ponatinib-induced PAH has been reported recently, suggesting that pulmonary vascular toxicity may be triggered by several TKIs [11]. As dasatinib, ponatinib and bosutinib are also known inhibitors of Src tyrosine kinase, it is conceivable that this pathway may be implicated in TKI-induced PAH. However, dasatinib-mediated apoptosis in pulmonary endothelial cells is not dependent of Src kinase inhibition [10]. In addition, bosutinib is a known potent inhibitor of fibroblast growth factor receptor and mitogen-activated protein kinases that are known signalling pathways involved in endothelial cell survival $[12,13]$, therefore we hypothesise that a potential mechanism could be the apoptosis of endothelial cells. As only a minority of patients exposed to TKIs will develop PAH, it is likely that other cofactors are required for the development of pleuro-pulmonary complications [14]. In the presented case reports it should be emphasised that the younger patients developed PAH while the older patients developed pleural effusions. Although this limited case series is insufficient to conclude an age effect, it is in accordance with the previous published reports of dasatinib-induced PAH where the median age was about 50 years old [3].

In conclusion, physicians should be aware of pulmonary complications of bosutinib therapy and of potential cross-intolerance with dasatinib. When unexplained dyspnoea occurs in patients treated with TKIs, chest radiography and transthoracic echocardiography should be performed in order to identify possible pleural or pulmonary vascular complications [15].

○ @ERSpublications

Pulmonary complications of bosutinib therapy and potential cross-intolerance with dasatinib http://ow.ly/UKWu303KGhx

Marianne Riou ${ }^{1,2,3,4}$, Andrei Seferian ${ }^{1,2,3}$, Laurent Savale ${ }^{1,2,3}$, Marie-Camille Chaumais ${ }^{1,3,5}$, Christophe Guignabert $\stackrel{1}{1,3}^{1,}$, Matthieu Canuet ${ }^{4}$, Pascal Magro ${ }^{6}$, Delphine Rea ${ }^{7}$, Olivier Sitbon ${ }^{1,2,3}$, Xavier Jaïs ${ }^{1,2,3}$, Marc Humbert ${ }^{1,2,3}$ and David Montani $i^{1,2,3}$

${ }^{1}$ Université Paris-Sud and Université Paris-Saclay, Le Kremlin-Bicêtre, France. ${ }^{2}$ AP-HP, Service de Pneumologie, Centre de Référence de l'Hypertension Pulmonaire Sévère, DHU Thorax Innovation, Hôpital Bicêtre, Le Kremlin-Bicêtre, France. ${ }^{3}$ INSERM UMR_S 999, Le Plessis Robinson, France. ${ }^{4}$ Service de Pneumologie, Nouvel Hôpital Civil, Hôpitaux Universitaires de Strasbourg, Strasbourg, France. ${ }^{5}$ AP-HP, Service de Pharmacie, DHU Thorax Innovation, Hôpital Antoine Béclère, Clamart, France. ${ }^{6}$ Service de Pneumologie, CHRU de Tours, Tours, France. ${ }^{7}$ AP-HP, Service d’Hématologie Adulte, Hôpital Saint-Louis, Paris, France.

Correspondence: David Montani, Service de Pneumologie, Centre de Référence de l'Hypertension Pulmonaire Sévère, Hôpital Bicêtre, 78 rue du Général Leclerc, 94270 Le Kremlin-Bicêtre, France. E-mail: david.montani@aphp.fr

Received: July 142016 | Accepted after revision: Aug 252016 | First published online: Oct 62016

Conflict of interest: Disclosures can be found alongside this article at erj.ersjournals.com

Acknowledgements: We acknowledge the French pulmonary hypertension pharmacovigilance network, VIGIAPATH, supported by the Agence Nationale de Sécurité du Médicament et des Produits de Santé (ANSM). 


\section{References}

1 O'Brien S, Radich JP, Abboud CN, et al. Chronic myelogenous leukemia, version 1.2015. J Natl Compr Canc Netw 2014; 12: 1590-1610.

2 Goldblatt M, Huggins JT, Doelken P, et al. Dasatinib-induced pleural effusions: a lymphatic network disorder? Am J Med Sci 2009; 338: 414-417.

3 Montani D, Bergot E, Gunther S, et al. Pulmonary arterial hypertension in patients treated by dasatinib. Circulation 2012; 125: 2128-2137.

4 Galie N, Humbert M, Vachiery J-L, et al. 2015 ESC/ERS Guidelines for the diagnosis and treatment of pulmonary hypertension: The Joint Task Force for the Diagnosis and Treatment of Pulmonary Hypertension of the European Society of Cardiology (ESC) and the European Respiratory Society (ERS): Endorsed by: Association for European Paediatric and Congenital Cardiology (AEPC), International Society for Heart and Lung Transplantation (ISHLT). Eur Respir J 2015; 46: 903-975.

5 Latagliata R, Breccia M, Fava C, et al. Incidence, risk factors and management of pleural effusions during dasatinib treatment in unselected elderly patients with chronic myelogenous leukaemia. Hematol Oncol 2013; 31: 103-109.

6 Shah NP, Rousselot P, Schiffer C, et al. Dasatinib in imatinib-resistant or -intolerant chronic-phase, chronic myeloid leukemia patients: 7-year follow-up of study CA180-034. Am J Hematol 2016; 91: 869-874.

7 Quintas-Cardama A, Kantarjian H, O'Brien S, et al. Pleural effusion in patients with chronic myelogenous leukemia treated with dasatinib after imatinib failure. J Clin Oncol 2007; 25: 3908-3914.

8 Gambacorti-Passerini C, Kantarjian HM, Kim D-W, et al. Long-term efficacy and safety of bosutinib in patients with advanced leukemia following resistance/intolerance to imatinib and other tyrosine kinase inhibitors. Am J Hematol 2015; 90: 755-768.

9 Kantarjian HM, Cortes JE, Kim D-W, et al. Bosutinib safety and management of toxicity in leukemia patients with resistance or intolerance to imatinib and other tyrosine kinase inhibitors. Blood 2014; 123: 1309-1318.

10 Guignabert C, Phan C, Seferian A, et al. Dasatinib induces lung vascular toxicity and predisposes to pulmonary hypertension. J Clin Invest 2016; 126: 3207-3218.

11 Quilot F-M, Georges M, Favrolt N, et al. Pulmonary hypertension associated with ponatinib therapy. Eur Respir J 2016; 47: 676-679.

$12 \mathrm{Tu}$ L, Dewachter L, Gore B, et al. Autocrine fibroblast growth factor-2 signaling contributes to altered endothelial phenotype in pulmonary hypertension. Am J Respir Cell Mol Biol 2011; 45: 311-322.

13 Remsing Rix LL, Rix U, Colinge J, et al. Global target profile of the kinase inhibitor bosutinib in primary chronic myeloid leukemia cells. Leukemia 2009; 23: 477-485.

14 Austin ED, Cogan JD, West JD, et al. Alterations in oestrogen metabolism: implications for higher penetrance of familial pulmonary arterial hypertension in females. Eur Respir J 2009; 34: 1093-1099.

15 Shepherd J. FDA drug safety podcast for healthcare professionals: Sprycel (dasatinib) and risk of pulmonary arterial hypertension. US Food and Drug Administration. www.fda.gov/Drugs/Drugsafety/DrugSafetyPodcasts/ ucm275517.htm Date last accessed: August 1, 2016 